\title{
THE MOST BASIC SELECTION CRITERIA FOR IMPROVING YIELD AND QUALITY OF UPLAND COTTON
}

\author{
Imtiaz ALI ${ }^{1^{*}}$, Amir SHAKEEL ${ }^{1}$, Asif SAEED ${ }^{l}$, Manzoor HUSSAIN ${ }^{2}$, Ahsan IRSHAD ${ }^{2}$, Muhammad Tariq \\ MAHMOOD ${ }^{3,}$ Zia Ullah ZIA ${ }^{1}$, Waqas MALIK ${ }^{4}$, Muhammad Kashif AZIZ ${ }^{2}$, Muhammad Arshad HUSSAIN ${ }^{2}$ \\ ${ }^{1}$ University of Agriculture, Department of Plant Breeding and Genetics, Faisalabad, PAKISTAN, \\ ${ }^{2}$ Regional Agricultural Research Institute, Bahawalpur, PAKISTAN, \\ ${ }^{3}$ Cotton Research Station, Bahawalpur, PAKISTAN, \\ ${ }^{4}$ Bahauddin Zakariya University, Faculty of Agricultural Science and Technology, Department of Plant \\ Breeding and Genetics, Multan, PAKISTAN \\ *Corresponding author: imtiaz.malghani@gmail.com
}

Received: 05.01.2016

\begin{abstract}
Increase in productivity and selection procedures chiefly depend upon genetic control of yield related traits, combining ability of the genotypes for concerned characters and association of these parameters among themselves and with the final yield. Single and three way crosses in cotton were analyzed for genetic behavior, combining ability, heterosis and correlation of various traits related to boll (synonymously known as withinboll yield traits) and fibre quality. The experiments were carried out at the Department of Plant Breeding and Genetics, University of Agriculture, Faisalabad. Crosses were made using line $\times$ tester mating design. All the genotypes showed highly significant variability with respect to all the parameters studied. Variances for both general combining ability (GCA) and specific combining ability (SCA) were significant for most of the traits however variances due to SCA were higher than variance due to GCA for all the traits. Likewise dominance variance $(D)$ was higher than additive variance $(A)$ which indicated dominant genetic behavior of the traits. The line BH-160 was found to be the best general combiner for most of the traits like average boll weight, number of seeds per boll, seed mass per boll, lint mass per boll, seed volume, seed surface area, seed cotton yield per plant and fibre fineness. Among testers, CIM-496 exhibited the highest GCA effects for boll weight, seed mass per boll, lint mass per boll and seed cotton yield per plant, while for number of seeds per boll, staple length and fibre strength, S-12 was considered the best. Highest SCA effects for boll weight, number of seeds per boll and seed mass per boll were displayed by (NIAB-228 $\times$ BH-160) $\times$ Reshmi-90. Most of the boll related traits revealed significant positive correlation with seed cotton yield.
\end{abstract}

Key words: Combining ability, correlation, Cotton, fibre quality, gene action, heterosis, within-boll yield components.

\section{INTRODUCTION}

Cotton is an important agricultural crop in the world which is grown for its fibre and oil in its seed (Bardak and Bolek, 2012). Among the four cultivated species of cotton, Gossypium hirsutum L., a domesticated tetraploid species accounts for more than $80 \%$ of the global cotton production (Shakeel et al., 2008). Pakistan is the $4^{\text {th }}$ major cotton producing and $5^{\text {th }}$ major cotton consuming country worldwide(Pakistan Economic survey 2012-13),however cotton productivity on per hectare basis is low i.e. $769 \mathrm{~kg}$ ha $^{-1}$ (Pakistan Economic survey 2012-13) as compared to many leading cotton producing countries of the world (USDA, FAO. 2013). Tremendous efforts have been made by plant breeders to increase cotton productivity but focus remained on direct selection for number of bolls on unit land area and boll size/weight for decades. Boll weight depends upon many within-boll yield contributing traits e.g. number, weight and volume of seeds, lint mass per boll and per seed, fibre length and number of fibre per unit seed surface area etc. A little selection has been made for these basic traits mainly because of the difficulty of their measurement.

Within-boll yield components are the most basic determinants of seed cotton and/or lint yield in cotton (Worley et al., 1974). According to Worley et al. (1974) the number of bolls on unit land area was the major contributor to seed cotton yield followed by lint mass per seed and number of seeds per boll. Among within-boll yield contributors, number of seeds of medium size and amount of lint produced on unit seed surface area were the main determinants of seed cotton yield (Ali et al., 2015). Culp and Harrell (1975) reported that increase in number 
of seeds per boll resulted in greater surface area per seed for greater lint production. Green and Culp (1990) determined significant general combining ability (GCA) for most of the fibre traits like fibre length, fibre uniformity, staple strength, seed cotton yield and lint percentage which suggested early generation selection to improve these traits. Good (GCA) effects for fibre fineness, fibre maturity, upper half mean length and fibre strength of fibre while negative GCA variances for most of the within-boll yield components were observed (Coyle \& Smith, 1997). Smith and Coyle (1997) determined negative correlation of Fibre length and fibre strength showed negative correlation with number of seeds per boll, seed surface area, number of spinnable fibre per unit seed surface area and lint percentage.

To check the assumption that plant population on unit land area could influence the within-boll yield components, Bednarz et al. (2006) conducted experiments regarding plant density modifications. They reported that plant density exhibited direct relation with total seed surface area per unit of land area but inverse relation with lint mass per boll, individual seed mass and seed number per boll. Bednarz et al. (2007) determined within-boll lint and seed parameters and fibre quality characters. They observed that the genotypes which had less seed size, produced more seed surface area but low lint mass and fibre number and vice versa. Seed size was the determinant of lint weight and number of fibre on unit seed surface area (Bednarz et al., 2007).

Higher estimates of SCA than GCA depicted the predominance of non-additive gene action for all traits except for fibre length, fibre strength and number of seeds per boll, which were additively controlled (Basal et al. 2009). They also reported negative correlation of fibre traits with the basic within-boll lint yield components. Tang and Xiao (2013) studied genetic affects and heterosis for within-boll yield components. Lint mass per seed, seed mass per seed and boll bur weight were primarily controlled by additive type of gene action and showed 39.6 to $58.9 \%$ heterosis. Lint mass per boll and number of seeds per boll displayed 36.4 to $48.4 \%$ increase over parents and exhibited dominance genetic effects. Ali et al. (2015) indicated preponderant effect of dominance variance in the expression of within-yield traits. They also reported involvement of inter-genic interactions in the phenotypic outcome of the traits.

Keeping in view the importance of most basic determinants of seed cotton yield and quality, the present study was aimed to analyze the combining ability effects and to estimate the range of heterotic potential for withinboll yield components and fibre quality traits.

\section{MATERIALS AND METHODS}

Research trials for the study were conducted at experimental area of the Department of Plant Breeding and Genetics, University of Agriculture, Faisalabad lies between longitude $73^{\circ}$ East, latitude $30.4^{\circ}$ North, with an elevation of 184 meters (604 ft) above sea level. Weather data regarding average temperature, total rainfall and relative humidity is shown in Figure 1. Three lines,NIAB228, BH-160and their $\mathrm{F}_{1}$ (NIAB-999 $\times$ BH-89)were crossed to ten testers namely CIM-70, Russian, CIM-496, CIM-1100, Reshmi-90, FH-634, S-12, NIAB-78, CIM240 and $\mathrm{VH}-144$, thus yielding 20 single and 10 three way crosses. Genotypes used as lines and testers originated from different breeding stations, methodology of evolution and possessed distinguishing characters with respect to plant morphology, seed cotton yield and fiber quality traits.

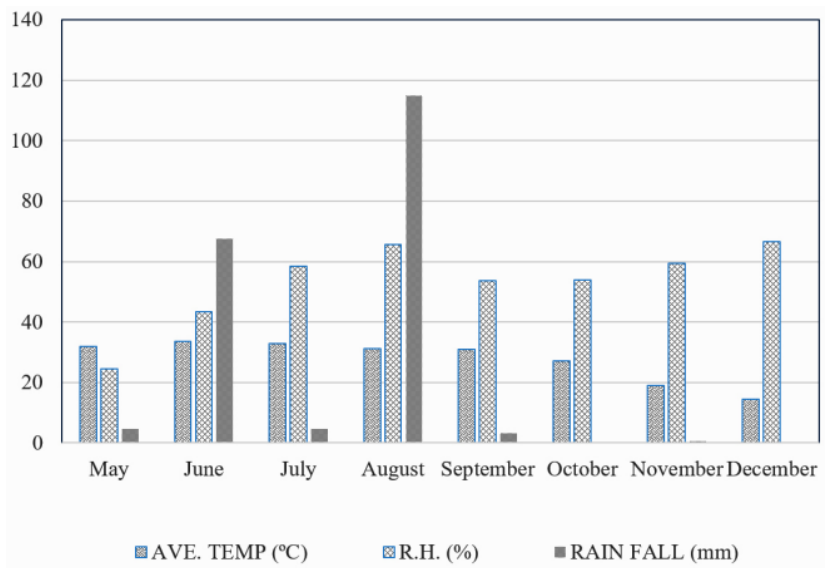

Figure 1. Weather data (average temperature, relative humidity, and rainfall) during the crop period.

The resulting line $\times$ tester progeny along with the parents were grown in field in three replicates using randomized complete block design. All the standard practices regarding irrigation, fertilization, weeding and plant protection measures were followed during the entire crop duration. A total of two pickings were made, one at $50 \%$ boll opening stage and the other at final maturity. Total bolls were counted and weighed from each of the 5 individual tagged plants in each plot from the two picks. A sample of three bolls, in each pick, was drawn from each of the 5 tagged plants, pooled for each plot and ginned. (Worley et al., 1976; Coyle \& Smith, 1997). The variables for which the data were recorded were as under:

No. of bolls/plant $(\mathrm{B} / \mathrm{P})=$ Total count of bolls picked from sampled plants.

Seed cotton yield/plant $(\mathrm{SC} / \mathrm{P})=$ Weight of total seed cotton from each sampled plant.

Average boll weight $(\mathrm{BW})=($ Seed cotton yield/plant $) /$ (No. of bolls/plant).

No. of seeds/boll $(\mathrm{S} / \mathrm{B})=($ No. of seeds in the sample/No. of bolls in the sample).

Seed mass/boll $(\mathrm{SM} / \mathrm{B})=($ Seed weight of the sample/No. of bolls in the sample).

Lint mass/boll $(\mathrm{LM} / \mathrm{B})=($ lint weight of the sample/No. of bolls in the sample).

Seed volume/100-seeds $=$ Measured by alcohol displacement method.

Seed density $=$ Weight of 100 seeds/Volume of 100 seeds. 
Surface area/seed $(\mathrm{SA} / \mathrm{S})=$ Seed volume was converted to surface area per seed by Hodson's (1920) table.

Fibre length, strength and fineness = Measured by automatic fibre testing machine (HVI-1100)

\section{Alcohol displacement method}

Seed volume was determined by alcohol displacement method. Alcohol is used due to low specific gravity and the seed dries out rapidly. For this purpose $50 \mathrm{ml}$ ethanol was taken in a graduated cylinder and a seed sample of 100 seeds was added to it. Total volume of ethanol and seeds was read directly from cylinder. Volume of 100 seeds was calculated by subtracting the volume of ethanol from total volume and expressed in $\mathrm{cm}^{3}$.

The data of various traits related to boll, seed cotton yield and fibre quality were subjected to line $\times$ tester analysis as suggested by Kempthorne (1957) to estimate the type of genetic control of the traits, general combining ability effects of parents and specific combining ability effects of crosses. Performance of crosses as compared to their respective better parents was also calculated to estimate the heterotic potential of studied genotypes for the studied traits. Correlation of various traits among themselves was analyzed using Pearson (1920) method.

\section{RESULTS}

Highly significant differences $(\mathrm{P} \geq 0.01)$ in genotypes, hybrids, parents, lines and testers were noted for all the traits indicating presence of considerable variability among the genotypes, however lines presented nonsignificant differences for fibre length (Table 1). Line $\times$ tester interaction was also found to be significant for all the characters. GCA variances were found to be nonsignificant while SCA variances were significant for all the traits. Some of the traits exhibited negative GCA variances including number of bolls per plant, seed volume, seed surface area, seed cotton per plant, fibre length and fibre strength. The variance due to SCA was higher than variance due to GCA for all the traits indicating the prevalence of non-additive genetic effects in the inheritance of these traits. Dominance variance (D) was found to be much higher than the additive (A) one. Degree of dominance, $\left(\sigma^{2} \mathrm{D} / \sigma^{2} \mathrm{~A}\right)^{1 / 2}$, was observed to be more than 1 for all the characters.

Table 1. Mean squares of various traits in cotton through line $\times$ tester analysis.

\begin{tabular}{|c|c|c|c|c|c|c|c|c|c|c|c|c|c|}
\hline $\mathrm{OV}$ & f. & $\mathbf{B} / \mathbf{P}$ & BW & S/B & M/B & LM/B & SV & SD & SSA & SC/P & FL & FST & FF \\
\hline Reps & 2 & 0.237 & 0.022 & 1.446 & 0.003 & 0.013 & 0.004 & 0.0003 & 0.0002 & 9.695 & 0.221 & 0.084 & 0.004 \\
\hline e & 42 & $14 * *$ & $0.472 * *$ & $08 * *$ & 3 *** & $0.231 * *$ & $1.110^{* *}$ & $0.029 * *$ & $12 * *$ & $392.676^{* *}$ & $4.016 * *$ & $13.579 * *$ & $44 * *$ \\
\hline & 12 & & & & & & & & & & & & \\
\hline & 29 & & & & & & & & & & & & \\
\hline & 1 & & & & & & & & & & & & \\
\hline & 2 & 2 & 1.460 & 12 & & & & & & & & & \\
\hline & 9 & & & & & & & & & & & & 0 \\
\hline & 18 & & & & & & & & & & & & \\
\hline Error & 84 & & & & & & & & & & & & \\
\hline Li11 & & & & & & & & & & & & & \\
\hline & & & & & & & & & & & & & \\
\hline $5^{2} \mathrm{GC}$ & & -0.0105 & & & & 0.0 & & & & & & & 0.010 \\
\hline $5^{2} \mathrm{~A}$ & & & & & & & & & & & & & 0.003 \\
\hline${ }^{2} \mathrm{D}$ & & 5.8564 & & & & & & & & & & & .165 \\
\hline${ }^{2} \mathrm{D}$ & & 6.917 & 21.465 & 8.153 & 10.770 & 8.155 & 6.827 & 9.798 & & 12.188 & 9.243 & 5.268 & 7.071 \\
\hline
\end{tabular}

B/P, number of bolls/plant; BW, average boll weight; S/B, number of seeds per boll; SM/B, seed mass/boll; LM/B, lint mass/boll; SV, seed volume per 100-seeds; SD, seed density; SSA, seed surface area; SC/P, seed cotton per plant; FL, fibre length; FF, fibre fineness; FST, fibre strength.

The female parent, BH-160 exhibited good GCA effects for most of the traits like boll weight, seeds per boll, seed mass per boll, lint mass per boll, seed volume, seed surface area, seed cotton per plant and fibre fineness. The other line, NIAB-228, was considered good general combiner for bolls per plant and seed density (Table 2). Among male parents CIM-496 showed the highest GCA effects for boll weight, seed mass per boll, lint mass per boll and seed cotton per plant. The other tester CIM-70 presented higher estimates of GCA effects for seed volume, seed surface area and fibre fineness.S-12 showed higher estimates of GCA effects for seeds per boll, fibre length and fibre strength while for FH-634 and CIM240were considered desirable genotypes for bolls per plant and seed density 
Table 2. General combining ability (GCA) effects of lines and testers for various traits in cotton.

\begin{tabular}{|c|c|c|c|c|c|c|c|c|c|c|c|c|}
\hline & $\mathbf{B} / \mathbf{P}$ & BW & $\mathbf{S} / \mathbf{B}$ & SM/B & LM/B & SV & SD & SSA & SC/P & FL & FST & FF \\
\hline \multicolumn{13}{|l|}{ Lines } \\
\hline NIAB-228 & $0.59 * *$ & $-0.13 * *$ & $-1.51 * *$ & $-0.04 * *$ & $-0.09 * *$ & $0.04 * *$ & $0.01 * *$ & $0.01 * *$ & $-0.87^{*}$ & -0.13 & $-0.31 *$ & $0.07 * *$ \\
\hline BH-160 & $0.35 * *$ & $0.25 * *$ & $2.35 * *$ & $0.10 * *$ & $0.16^{* *}$ & $0.16 * *$ & $-0.02 * *$ & $0.02 * *$ & $5.88 * *$ & -0.05 & 0.22 & $-0.22 * *$ \\
\hline NIAB-228 $\times$ BH-160 & $-0.94 * *$ & $-0.12 * *$ & $-0.84 * *$ & $-0.06 * *$ & $-0.06^{* *}$ & $-0.20 * *$ & $0.01 * *$ & $-0.02 * *$ & $-5.02 * *$ & 0.18 & 0.09 & $0.16^{* *}$ \\
\hline S.E. Lines & 0.13 & 0.014 & 0.179 & 0.012 & 0.011 & 0.015 & 0.003 & 0.002 & 0.423 & 0.131 & 0.131 & 0.018 \\
\hline \multicolumn{13}{|l|}{ Testers } \\
\hline CIM-70 & 0.23 & 0.03 & $2.19 * *$ & $0.20 * *$ & $-0.17 * *$ & $0.33 * *$ & $-0.06 * *$ & $0.03 * *$ & $1.65^{*}$ & -0.11 & 0.33 & $0.38 * *$ \\
\hline Russian & $-0.81 * *$ & $-0.36^{* *}$ & $-2.88 * *$ & $-0.19 * *$ & $-0.17 * *$ & $0.13 * *$ & $-0.05 * *$ & $0.01 * *$ & $-9.00 * *$ & 0.17 & 0.27 & $0.36 * *$ \\
\hline CIM-496 & -0.01 & $0.47 * *$ & $2.31 * *$ & $0.26 * *$ & $0.20 * *$ & $0.09 * *$ & $0.05^{* *}$ & $0.01 * *$ & $7.80 * *$ & -0.34 & $0.61 *$ & $-0.30 * *$ \\
\hline CIM-1100 & $-0.52 *$ & $-0.15 * *$ & $-1.42 * *$ & $-0.09 * *$ & $-0.06 * *$ & 0.00 & $-0.06 * *$ & 0.00 & $-4.70 * *$ & -0.04 & $-0.93 * *$ & $0.19 * *$ \\
\hline Reshmi-90 & $-1.34 * *$ & $-0.09 * *$ & -0.61 & $0.07 * *$ & $-0.16^{* *}$ & $-0.12 * *$ & $0.04 * *$ & $-0.01 * *$ & $-6.13 * *$ & -0.04 & $-1.26^{* *}$ & $-0.10 * *$ \\
\hline FH-634 & $1.57 * *$ & -0.04 & $-3.35 * *$ & $-0.17 * *$ & $0.13 * *$ & $0.27 * *$ & $0.05^{* *}$ & $0.03 * *$ & $4.28 * *$ & $-0.56^{*}$ & $0.60 *$ & $-0.26^{* *}$ \\
\hline S-12 & 0.08 & 0.03 & $4.51 * *$ & $0.17 * *$ & $-0.14 * *$ & $-0.11 * *$ & $-0.06 * *$ & $-0.01 * *$ & 0.94 & $1.06 * *$ & $1.53 * *$ & 0.06 \\
\hline NIAB-78 & -0.06 & $0.10^{* *}$ & $2.09 * *$ & 0.00 & $0.10 * *$ & $-0.39 * *$ & -0.00 & $-0.04 * *$ & 1.15 & $0.59 *$ & -0.35 & $-0.07^{*}$ \\
\hline CIM-240 & -0.41 & -0.03 & $-2.89 * *$ & $-0.21 * *$ & $0.18 * *$ & $-0.15^{* *}$ & $0.11 * *$ & $-0.02 * *$ & -1.21 & 0.33 & $-1.77 * *$ & $-0.39 * *$ \\
\hline VH-144 & $1.28 * *$ & 0.04 & 0.06 & $-0.05 *$ & $0.09 * *$ & -0.05 & $-0.01 *$ & -0.00 & $5.22 * *$ & $-1.05 * *$ & $0.97 * *$ & $0.13 * *$ \\
\hline S.E. Testers & 0.238 & 0.026 & 0.326 & 0.022 & 0.021 & 0.026 & 0.006 & 0.003 & 0.773 & 0.238 & 0.239 & 0.032 \\
\hline
\end{tabular}

B/P, number of bolls/plant; BW, average boll weight; S/B, number of seeds per boll; SM/B, seed mass/boll; LM/B, lint mass/boll; SV, seed volume per 100-seeds; SD, seed density; SSA, seed surface area; SC/P, seed cotton per plant; FL, fibre length; FF, fibre fineness; FST, fibre strength.

Table 3. Specific combining ability (SCA) effects of single and three way crosses for various traits in cotton.

\begin{tabular}{|c|c|c|c|c|c|c|c|c|c|c|c|c|}
\hline Cross & $\mathbf{B} / \mathbf{P}$ & BW & S/B & SM/B & LM/B & SV & SD & SSA & SC/P & FL & FST & FF \\
\hline NIAB-228 × CIM-70 & $-1.10 * *$ & $-0.16^{* *}$ & $-1.40^{*}$ & $-0.09 *$ & $-0.08^{*}$ & $0.12 * *$ & -0.00 & $0.01 * *$ & $-6.50 * *$ & -0.05 & 0.65 & $0.41 * *$ \\
\hline NIAB-228 $\times$ Russian & -0.06 & $-0.37 * *$ & $-4.40 * *$ & $-0.27 * *$ & $-0.10 * *$ & 0.06 & 0.01 & 0.01 & $-6.38 * *$ & $-1.03 *$ & $3.68 * *$ & $0.37 * *$ \\
\hline NIAB-228 × CIM-496 & $-4.06 * *$ & $0.27 * *$ & $3.63 * *$ & $0.19 * *$ & $0.08^{*}$ & $0.29 * *$ & $-0.10^{* *}$ & $0.03 * *$ & $-9.18 * *$ & 0.12 & 0.34 & -0.04 \\
\hline NIAB-228 $\times$ CIM-1100 & $2.39 * *$ & $0.41^{* *}$ & $2.41 * *$ & $0.15 * *$ & $0.25 * *$ & $-0.15 * *$ & $0.04 * *$ & $-0.02 * *$ & $15.55^{* *}$ & -0.12 & $-3.26^{* *}$ & -0.10 \\
\hline NIAB-228 $\times$ Reshmi-90 & 0.81 & 0.03 & -0.87 & $-0.10^{* *}$ & $0.13 * *$ & $0.47 * *$ & $-0.07 * *$ & $0.05 * *$ & $3.52^{* *}$ & 0.42 & 0.44 & $-0.14 *$ \\
\hline NIAB-228 × FH-634 & $-1.83 * *$ & $-0.61 * *$ & $-5.89 * *$ & $-0.41 * *$ & $-0.20 * *$ & $0.11^{*}$ & $0.02 *$ & $0.01 *$ & $-16.76^{* *}$ & -0.13 & $1.22 * *$ & $-0.29 * *$ \\
\hline NIAB-228 $\times$ S-12 & $1.79 * *$ & $0.44 * *$ & $1.63 * *$ & $0.32 * *$ & $0.12 * *$ & $-1.18 * *$ & $0.22 * *$ & $-0.12 * *$ & $14.08 * *$ & -0.82 & $-4.71 * *$ & $-0.13^{*}$ \\
\hline$-228 \times$ NIAB-78 & $2.32 * *$ & $-0.18 * *$ & $3.52 * *$ & 0.01 & $-0.19 * *$ & 0.04 & $-0.13 * *$ & 0.00 & $4.10^{* *}$ & $0.85^{*}$ & $0.87 *$ & -0.08 \\
\hline NIAB-228 × CIM-240 & $-1.19 * *$ & 0.07 & $-1.44 *$ & -0.00 & $0.08 *$ & $0.64 * *$ & $-0.04 * *$ & $0.06^{* *}$ & $-2.70^{*}$ & -0.65 & $3.35^{* *}$ & $-0.19 * *$ \\
\hline NIAB-228 $\times$ VH-144 & $0.92 *$ & $0.11^{*}$ & $2.81 * *$ & $0.20 * *$ & $-0.10^{* *}$ & $-0.40 * *$ & $0.03 *$ & $-0.04 * *$ & $4.27 * *$ & $1.40^{* *}$ & $-2.59 * *$ & $0.19 * *$ \\
\hline CIM-70 & $2.47 * *$ & $0.21 * *$ & $1.44 *$ & $0.13 * *$ & $0.08^{*}$ & $-0.56^{* *}$ & $0.05 * *$ & $-0.06^{* *}$ & $12.02 * *$ & 0.30 & -1 & 0.10 \\
\hline BH-16 & $-0.82 *$ & $0.30^{* *}$ & 0.27 & $0.21^{* *}$ & $0.09 *$ & $0.28 * *$ & $0.04 * *$ & $0.03 * *$ & 2.20 & -0.21 & $-1.05^{*}$ & 0.05 \\
\hline BH-160 × CIM-496 & $1.58 * *$ & $-0.17 * *$ & $-4.27 * *$ & $-0.26^{* *}$ & $0.09 *$ & $-0.12 * *$ & $0.09 * *$ & $-0.01 *$ & $2.77 *$ & -0.40 & 0.45 & -0.02 \\
\hline 00 & $-4.10 * *$ & $0.11^{*}$ & 0.47 & $0.10^{*}$ & 0.01 & 0.03 & -0.01 & 0.00 & $-11.73 * *$ & 0.44 & $2.08 * *$ & -0.08 \\
\hline-90 & 0.05 & $-0.58 * *$ & $-7.16^{* *}$ & $-0.38 * *$ & $-0.21 * *$ & $0.39 * *$ & $-0.03 *$ & $0.04 * *$ & $-9.86^{* *}$ & $-1.43 * *$ & $1.25 * *$ & $-0.39 * *$ \\
\hline$\times$ FH-634 & $1.54 * *$ & 0.07 & $3.62 * *$ & $0.30 * *$ & $-0.23 * *$ & $-0.40 * *$ & $-0.05 * *$ & $-0.04 * *$ & $6.29 * *$ & $0.93^{*}$ & 0.32 & $-0.30^{* *}$ \\
\hline$\times \mathrm{S}-12$ & 0.03 & $-0.37 * *$ & $1.30^{*}$ & $-0.11 * *$ & $-0.26^{* *}$ & $0.41 * *$ & $-0.13 * *$ & $0.04 * *$ & $-7.14 * *$ & -0.70 & $2.66 * *$ & -0.05 \\
\hline$\times$ NIAB -78 & $-3.77 * *$ & $-0.23 * *$ & -0.29 & -0.06 & $-0.16^{* *}$ & $-0.51 * *$ & $-0.02 *$ & $-0.05^{* *}$ & $-16.18 * *$ & 0.40 & $-2.50 * *$ & $-0.26 * *$ \\
\hline BH-IC & $1.85^{* *}$ & $0.37 * *$ & $2.22 * *$ & 0.03 & $0.34 * *$ & 0.06 & $0.08 * *$ & 0.01 & $12.28 * *$ & 0.00 & $-2.81 * *$ & $0.60 * *$ \\
\hline BH-160 × VH-144 & $1.16^{* *}$ & $0.29 * *$ & $2.42 * *$ & 0.04 & $0.25 * *$ & $0.42 * *$ & -0.0 & $0.04 * *$ & $9.35 * *$ & 0.65 & 0.68 & $0.34 * *$ \\
\hline $8 \times \mathrm{BH}-160) \times$ & $-1.37 * *$ & -0.05 & -0.04 & -0.04 & -0.01 & $0.43 * *$ & $-0.05 * *$ & $0.04 * *$ & $-5.51 * *$ & -0.26 & 0.42 & $-0.51 *$ \\
\hline$(\mathrm{NIAB}-228 \times \mathrm{BH}-160) \times$ & $0.87 *$ & 0.07 & $4.13 * *$ & 0.06 & 0.01 & $-0.34 * *$ & $-0.05 * *$ & $-0.03 * *$ & $4.17 * *$ & $1.23 * *$ & $-2.62 * *$ & $-0.42 * *$ \\
\hline$(\mathrm{NIAB}-228 \times \mathrm{BH}-160) \times \mathrm{CIM}-496$ & $2.47 * *$ & $-0.10^{*}$ & 0.65 & 0.07 & $-0.17 * *$ & $-0.17 * *$ & 0.00 & $-0.02 * *$ & $6.41 * *$ & 0.28 & -0.79 & 0.07 \\
\hline 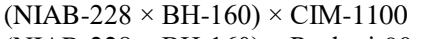 & $1.72 * *$ & $-0.51 * *$ & $-2.88^{* *}$ & $-0.25 *$ & $-0.26^{* *}$ & $0.12^{*}$ & $-0.03 * *$ & $0.01 *$ & $-3.83 * *$ & -0.32 & $1.18 * *$ & $0.18^{* *}$ \\
\hline$-228 \times B$ & $-0.86^{*}$ & $0.56^{* *}$ & $8.03 * *$ & $0.48 * *$ & $0.08^{*}$ & $-0.86^{* *}$ & $0.09 * *$ & $-0.09 * *$ & $6.34 * *$ & $1.01 *$ & $-1.69 * *$ & $0.53 * *$ \\
\hline$(\mathrm{NIAB}-228 \times \mathrm{BH}-160) \times \mathrm{FH}-634$ & 0.29 & $0.54 * *$ & $2.27 * *$ & $0.11 * *$ & $0.43 * *$ & $0.29 * *$ & $0.02 *$ & $0.03 * *$ & $10.46^{* *}$ & -0.80 & $-1.55^{* *}$ & $0.59 * *$ \\
\hline$(\mathrm{NIAB}-228 \times \mathrm{BH}-160) \times$ & $-1.82 * *$ & -0.07 & $-2.93 * *$ & $-0.21 * *$ & $0.14 * *$ & $0.76^{* *}$ & $-0.10^{* *}$ & $0.08 * *$ & $-6.94 * *$ & $1.51^{* *}$ & $2.05 * *$ & $0.18^{* *}$ \\
\hline AB-78 & $1.45^{* *}$ & $0.41^{* *}$ & $-3.22 * *$ & 0.05 & $0.35 * *$ & $0.48 * *$ & $0.15 * *$ & $0.05^{* *}$ & $12.09 * *$ & $-1.26 * *$ & $1.63 * *$ & $0.33^{* *}$ \\
\hline B- $228 \times$ BH-160) $\times$ CIM-240 & -0.66 & $-0.44 * *$ & -0.78 & -0.03 & $-0.41 * *$ & $-0.69 * *$ & $-0.04 * *$ & $-0.07 * *$ & $-9.58 * *$ & 0.64 & -0.55 & $-0.41 * *$ \\
\hline$(\mathrm{NIAB}-228 \times \mathrm{BH}-160) \times \mathrm{VH}-1$ & $-2.08 * *$ & $-0.40^{* *}$ & $-5.22 * *$ & $-0.25 * *$ & $-0.15^{* *}$ & -0.02 & 0.00 & -0.00 & $-13.61 * *$ & $-2.04 * *$ & $1.91 * *$ & $-0.53 * *$ \\
\hline S.E. & 0.412 & 0.046 & 0.565 & 0.038 & 0.036 & 0.046 & 0.01 & 0.005 & 1.338 & 0.413 & 0.414 & 0.056 \\
\hline
\end{tabular}

The three way cross $($ NIAB-228 $\times$ BH-160) $\times$ Reshmi90 produced the highest SCA effects regarding boll weight, seeds per boll and seed mass per boll while for the traits like seed volume, seed surface area and fibre length the three way cross $(\mathrm{NIAB}-228 \times \mathrm{BH}-160) \times \mathrm{S}-12$ was considered the most favorable (Table 3). For boll per plant, the single cross $\mathrm{BH}-160 \times \mathrm{CIM}-70$ and the three way cross $(\mathrm{NIAB}-228 \times \mathrm{BH}-160) \times \mathrm{CIM}-496$ displayed the highest SCA effects equal in magnitude (2.47). The cross combinations NIAB-228 $\times$ S-12, NIAB-228 $\times$ CIM1100 and NIAB-228 $\times$ Russian exhibited the maximum SCA effects for seed density, seed cotton per plant and fibre strength, respectively.

Contribution of line $\times$ tester interaction, to the total variability, was higher as compared to lines or testers individual contribution for all the characters (Figure 2). Similarly if we compare contribution of lines and testers, the testers contributed more for all the traits. 


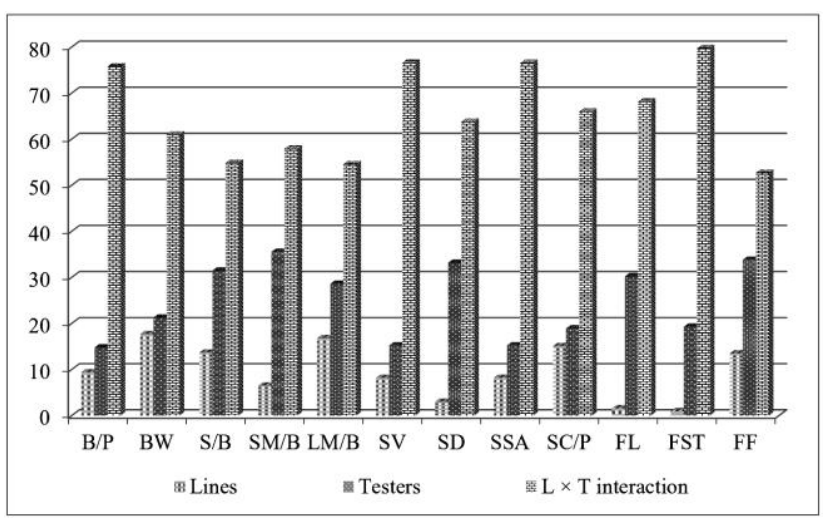

Figure 2.Proportional contribution of lines, testers and $\mathrm{L} \times \mathrm{T}$ interaction

$\mathrm{B} / \mathrm{P}$, number of bolls/plant; BW, average boll weight; $\mathrm{S} / \mathrm{B}$, number of seeds per boll; SM/B, seed mass/boll; LM/B, lint mass/boll; SV, seed volume per 100-seeds; SD, seed density; SSA, seed surface area; SC/P, seed cotton per plant; FL, fibre length; FST, fibre strength; FF, fibre fineness.
Results pertaining to heterosis, as compared to respective better parent, in various traits, revealed that the single cross NIAB-228 $\times$ NIAB-78 showed the highest value for lint mass per boll $(26.84 \%)$, seed cotton per plant $(23.40 \%)$ and a low range of heterosis was observed for fibre length $(4.40 \%)$. Maximum heterosis for average boll weight $(16.31 \%)$ and seed density $(5.03 \%)$, which is in a low range, was observed for the cross NIAB-228 $\times$ S12 while NIAB-228 $\times$ Reshmi-90 showed an increase of $27.23 \%$ and $27.24 \%$ over the better parent for seed volume per 100 seeds and seed surface area. The single crosses i.e. NIAB-228 $\times$ CIM-70 and $\mathrm{BH}-160 \times$ NIAB-78 produced $10.97 \%$ and $23.18 \%$ more bolls per plant and seeds per boll, respectively, as compared to respective better parent. Three way crosses could not perform well regarding heterosis over better parent, only two crosses i.e. $(\mathrm{NIAB}-228 \times \mathrm{BH}-160) \times \mathrm{S}-12$ and $(\mathrm{NIAB}-228 \times \mathrm{BH}-$ $160) \times$ Reshmi-90could gain the highest position for fibre strength and fibre fineness, respectively (Table 4).

Table 4. Heterotic effects, as over the better parent, of single and three way crosses for various traits in cotton.

\begin{tabular}{|c|c|c|c|c|c|c|c|c|c|c|c|c|}
\hline Cross & $\mathbf{B} / \mathbf{P}$ & BW & S/B & SM/B & LM/B & SV & SD & SSA & SC/P & FL & FST & FF \\
\hline NIAB-228 × CIM-70 & $-20.30^{* *}$ & $-9.51 * *$ & -4.64 & $10.76 * *$ & $-39.68 * *$ & $14.95 * *$ & $-17.64 * *$ & $15.00 * *$ & $-27.95 * *$ & $-7.67 * *$ & 2.09 & $3.66^{* *}$ \\
\hline NIAB-228 × Russian & $10.97 * *$ & $-26.83 * *$ & $-40.66^{* *}$ & $-25.82 * *$ & $-28.70 * *$ & $18.41 * *$ & $-15.94 * *$ & $18.35^{* *}$ & $-19.11 * *$ & $-5.22 *$ & $9.85 * *$ & 2.44 \\
\hline NIAB-228 × CIM-496 & $-21.53 * *$ & $15.78^{* *}$ & $14.81 * *$ & $30.67 * *$ & -5.02 & $3.39 * *$ & $-17.06 * *$ & $3.37 * *$ & $-9.20 * *$ & $-8.97 * *$ & $5.14 *$ & $-17.07 * *$ \\
\hline NIAB-228 × CIM-1100 & 0.66 & $7.46^{* *}$ & 1.17 & 5.51 & $10.42 * *$ & 0.44 & $-13.66^{* *}$ & 0.48 & $8.19 *$ & -3.93 & $-19.78 * *$ & $-9.15 * *$ \\
\hline NIAB-228 $\times$ Reshmi-90 & 4.69 & $-7.85 * *$ & $-34.00 * *$ & $-15.07 * *$ & 5.86 & $27.23 * *$ & $-15.32 * *$ & $27.24 * *$ & -3.48 & -1.84 & -0.00 & $-15.24 * *$ \\
\hline NIAB-228 × FH-634 & 4.21 & $-23.85 * *$ & $-39.35^{* *}$ & $-38.45^{* *}$ & 2.46 & $10.91 * *$ & $-4.15 * *$ & $10.88 * *$ & $-20.61 * *$ & $-7.70 * *$ & $6.59 * *$ & $-20.73 * *$ \\
\hline NIAB-228 $\times$ S-12 & 3.75 & $16.31 * *$ & 4.61 & $23.06 * *$ & 5.08 & $-14.16^{* *}$ & $5.03 * *$ & $-14.16^{* *}$ & $20.71 * *$ & $-4.22 *$ & $-8.59 * *$ & $-12.20^{* *}$ \\
\hline NIAB-228 $\times$ NIAB-78 & $7.27 *$ & $13.82 * *$ & $20.71 * *$ & 5.00 & $26.84 * *$ & $-9.76^{* *}$ & $-25.14 * *$ & $-9.81 * *$ & $23.40 * *$ & $4.40 *$ & $-4.41 *$ & $-13.41 * *$ \\
\hline NIAB-228 $\times$ CIM-240 & $-13.10^{* *}$ & $-15.65^{* *}$ & $-34.61 * *$ & $-25.46^{* *}$ & -1.55 & $19.90 * *$ & $-4.96 * *$ & $19.96 * *$ & $-26.66^{* *}$ & $-5.81 * *$ & $8.71 * *$ & $-21.34 * *$ \\
\hline NIAB- $228 \times V$ & 0.98 & $11.91 * *$ & 2.23 & $13.37 * *$ & 5.65 & $14.66^{* *}$ & $-10.60 * *$ & $14.70 * *$ & $13.05 * *$ & -0.87 & -2.90 & $-4.88 * *$ \\
\hline $\mathrm{BH}-$ & -5.15 & $11.75 * *$ & 17.89 & 42.2 & $-18.85^{* *}$ & $-6.53 * *$ & -16.7 & $-6.53 * *$ & $8.83 * *$ & $-9.13 * *$ & $-7.75^{* *}$ & $17.83 * *$ \\
\hline BH-160 $\times \mathrm{Ru}$ & $-20.26^{* *}$ & 2.39 & $-15.67 * *$ & 8.9 & $-18.16^{* *}$ & 1.22 & $-18.15^{* *}$ & 1.24 & $-18.35 * *$ & $-9.93 * *$ & $-7.86^{* *}$ & $16.28 * *$ \\
\hline BH-160 $\times$ CIN & -4.82 & $13.31 * *$ & 0.95 & 15.8 & 3.87 & $-4.08^{* *}$ & -2 & $-4.08 * *$ & $7.77 *$ & $-12.33 * *$ & -1.86 & -0.78 \\
\hline BH-160 $\times$ CIM & -34.7 & 3.01 & 7.96 & $10 .($ & -16. & $-3.27 * *$ & -23 . & $-3.28 * *$ & -32 & $-8.45^{* *}$ & -1 & $9.30 * *$ \\
\hline BH-1 & -18 & -16.6 & -40.1 & -21 & -35 . & -0 & -15 & -0 & -32 & $-14.84 * *$ & -5.3 & $4.65^{* *}$ \\
\hline BH- & 2.57 & 5.2 & 4.9 & 4 & $-19.71 * *$ & $-5.31^{* *}$ & -15.6 & $-5.29 * *$ & $7.77^{*}$ & $-8.56^{* *}$ & -2.29 & $-6.20 * *$ \\
\hline BH-160 × S-12 & $-11.90 * *$ & $-6.24 * *$ & $15.54 * *$ & $6.99 *$ & $-37.21 * *$ & -0.00 & $-35.25 * *$ & 0.00 & $-17.44 * *$ & $-8.56^{* *}$ & $8.41^{* *}$ & $6.98 * *$ \\
\hline BH-160 × NIAB-78 & $-30.87 * *$ & 0.31 & $23.18 * *$ & $12.18^{* *}$ & $-17.51 * *$ & $-15.04 * *$ & $-19.39 * *$ & $-15.11 * *$ & $-30.73 * *$ & $-6.39 * *$ & $-14.63 * *$ & -0.78 \\
\hline BH-160 × CIM-240 & -5.47 & 3.56 & $-13.46^{* *}$ & $-17.36^{* *}$ & $18.06^{* *}$ & $-4.90 * *$ & 2.02 & $-4.89 * *$ & 4.94 & $-8.68 * *$ & $-20.31 * *$ & $11.63 * *$ \\
\hline BH-160 × VH-144 & -0.64 & $14.55 * *$ & $13.88 * *$ & $14.62 * *$ & $6.86^{*}$ & 0.82 & $-20.56 * *$ & 0.84 & $13.78 * *$ & $-11.19 * *$ & 0.11 & $17.83^{* *}$ \\
\hline$($ NIAB-228 $\times$ BH-160) $\times$ CIM-70 & $-33.33 * *$ & $-13.53 * *$ & 2.19 & $12.33 * *$ & $-39.23 * *$ & $15.89 * *$ & $-29.35 * *$ & $15.96 * *$ & $-42.20 * *$ & $-8.39 * *$ & 2.67 & $13.28 * *$ \\
\hline$(\mathrm{NIAB}-228 \times \mathrm{BH}-160) \times \mathrm{R}$ & $-28.25 * *$ & $-21.27 * *$ & $-13.69 * *$ & $-8.01 *$ & $-38.17 * *$ & $2.34 * *$ & $-28.94 * *$ & $2.39 * *$ & $-43.41 * *$ & -2.21 & $-10.19 * *$ & $14.84 * *$ \\
\hline $\mathrm{AB}-228 \times \mathrm{BH}-160) \times \mathrm{C}$ & $-18.08 * *$ & -1.96 & $6.87 *$ & $22.91 * *$ & $-26.79 * *$ & $-5.51^{* *}$ & $-14.61 * *$ & $-5.53 * *$ & $-19.67 * *$ & $-7.34 * *$ & 2.02 & $10.94 * *$ \\
\hline $\mathrm{JIAB}-228 \times \mathrm{BH}-160) \times \mathrm{C}$ & $-23.45 * *$ & $-32.16^{* *}$ & $-15.24 * *$ & $-18.62 * *$ & $-47.82 * *$ & 0.88 & $-27.44 * *$ & 0.91 & $-48.02 * *$ & $-8.39 * *$ & $-3.76^{*}$ & $25.00 * *$ \\
\hline$(\mathrm{NIAB}-228 \times \mathrm{BH}-160) \times \mathrm{R}$ & $-37.85^{* *}$ & 1.08 & $-9.79 * *$ & $11.89 * *$ & $-33.47 * *$ & $-8.41^{* *}$ & $-7.06 * *$ & $-8.39 * *$ & $-37.13 * *$ & -3.73 & $-7.85^{* *}$ & $26.56 * *$ \\
\hline$($ NIAB-228 $\times$ BH-160) $\times$ FH-634 & $-20.62 * *$ & 1.96 & $-10.09 * *$ & $-12.92 * *$ & 4.21 & $10.00 * *$ & $-12.18 * *$ & $9.95 * *$ & $-19.00 * *$ & $-11.89 * *$ & -1.76 & $24.22 * *$ \\
\hline$(\mathrm{NIAB}-228 \times \mathrm{BH}-160) \times \mathrm{S}-12$ & $-35.88 * *$ & $-14.02 * *$ & $-7.50^{* *}$ & $-6.99 *$ & $-28.68 * *$ & $8.41 * *$ & $-33.72 * *$ & $8.47 * *$ & $-44.86 * *$ & 1.86 & $15.46 * *$ & $21.87 * *$ \\
\hline AB-228 $\times$ BH-160) $\times$ NIAB-78 & $-22.60 * *$ & 2.16 & -1.88 & 6.54 & -2.22 & $-7.32^{* *}$ & $-5.40 * *$ & $-7.38^{* *}$ & $-20.87 * *$ & $-9.44 * *$ & -0.55 & $22.66 * *$ \\
\hline$($ NIAB-228 $\times$ BH-160) $\times$ CIM-240 & $-33.05^{* *}$ & $-29.99 * *$ & $-30.89 * *$ & $-27.79 * *$ & $-42.33 * *$ & $-6.54 * *$ & $-13.33 * *$ & $-6.47 * *$ & $-50.85 * *$ & -3.73 & $-5.59 * *$ & -2.34 \\
\hline$($ NIAB-228 $\times$ BH-160) $\times$ VH-144 & $-31.92 * *$ & $-23.53 * *$ & $-22.53 * *$ & $-14.25 * *$ & $-32.74 * *$ & $4.21 * *$ & $-20.14 * *$ & $4.22 * *$ & $-47.86^{* *}$ & $-17.95 * *$ & $12.96 * *$ & $7.03 * *$ \\
\hline
\end{tabular}

Critical analysis of correlation coefficients of various traits revealed that the number of bolls per plant showed significant positive correlation with seed cotton yield (81.8\%), fibre length $(72.5 \%)$ and fibre strength $(46.5 \%)$ while negatively correlated with seed mass per boll, seed density and fibre fineness (Table 5). Boll weight was also highly correlated with seed cotton yield and seed number per boll. Seed number per boll exhibited negative association with seed density, lint mass per boll and micronaire value of the fibre. Seed mass per boll had direct relationship with seed density and lint mass per boll while negative association with fibre length and strength. Total surface area of seeds per boll and lint mass per boll were positively correlated. Relationship of seed density was parallel with lint mass per boll and fibre fineness while anti-parallel with fibre length and fibre strength. Fibre length and fibre fineness showed negative correlation with one another, same was the case between fibre fineness and fibre strength. 
Table 5. Correlation coefficients among various traits in cotton.

\begin{tabular}{|c|c|c|c|c|c|c|c|c|c|c|c|}
\hline & B/P & BW & S/B & SM/B & SSA & SV & SD & LM/B & FL & FF & FST \\
\hline BW & -0.227 & & & & & & & & & & \\
\hline S/B & $-0.315^{*}$ & $0.673 * *$ & & & & & & & & & \\
\hline $\mathrm{SM} / \mathrm{B}$ & $-0.671 * *$ & -0.038 & $0.317 *$ & & & & & & & & \\
\hline SSA & -0.014 & 0.005 & $-0.368 *$ & -0.215 & & & & & & & \\
\hline SV & 0.093 & 0.057 & -0.179 & -0.219 & $1.000 * *$ & & & & & & \\
\hline SD & $-0.727 * *$ & $-0.356^{*}$ & $-0.683^{* *}$ & $0.886 * *$ & $-0.344 *$ & -0.167 & & & & & \\
\hline LM/B & $-0.679 * *$ & $0.305^{*}$ & $-0.631 * *$ & $0.882 * *$ & $0.701 * *$ & -0.088 & $0.979 * *$ & & & & \\
\hline FL & $0.725^{* *}$ & $0.413 * *$ & $0.700 * *$ & $-0.755^{* *}$ & -0.175 & 0.036 & $-0.871 * *$ & $-0.835 * *$ & & & \\
\hline FF & $-0.750 * *$ & $-0.396 * *$ & $-0.622 * *$ & $0.865^{* *}$ & -0.057 & -0.148 & $0.949 * *$ & $0.916^{* *}$ & $-0.865 * *$ & & \\
\hline FST & $0.465 * *$ & 0.097 & $0.305^{*}$ & $-0.720 * *$ & $0.569 * *$ & $0.500 * *$ & $-0.715 * *$ & $-0.692 * *$ & $0.545^{* *}$ & $-0.675^{* *}$ & \\
\hline SCY & $0.818 * *$ & $0.808 * *$ & $0.569 * *$ & $0.428 * *$ & -0.011 & 0.056 & 0.238 & $0.315^{*}$ & $0.515 * *$ & $-0.498 * *$ & 0.181 \\
\hline
\end{tabular}

B/P, number of bolls/plant; BW, average boll weight; S/B, number of seeds per boll; SM/B, seed mass/boll; LM/B, lint mass/boll; SV, seed volume per 100-seeds; SD, seed density; SSA, seed surface area; SC/P, seed cotton per plant; FL, fiber length; FF, fiber fineness; FST, fiber strength.

\section{DISCUSSION}

Highly significant differences among genotypes, hybrids, parents, lines and testers indicated presence of considerable variability in the genetic material. Male and female parents each originated from different breeding institutes and method of evolution presented significant differences among themselves. Non-significant GCA variances while significant SCA variances indicated the key role of non-additive type of genes controlling the expression of all the traits. Some of the traits exhibited negative GCA variances including number of bolls per plant, seed volume, seed surface area, seed cotton per plant, fibre length and fibre strength which may be due to the insufficient number of lines and testers used. Male and female parents were kept limited due to load of work, lack of facilities and time limit for the research project. Coyle and Smith (1997) reported negative GCA variances for basic within-boll yield components. Negative variances may be attributed to environmental influences i.e. higher inter-replication differences. Preetha and Raveendren (2008); Pole et al. (2008); Swamy et al. (2013) reported that both additive and non-additive variances were important. The variance due to SCA was higher than variance due to GCA for all the traits indicating the prevalence of non-additive genetic effects in the inheritance of these traits. Dominance variance (D) was found to be much higher than the additive (A) one which strongly favors the heterosis breeding or late generation selection for improvement of these characters. Degree of dominance, $\left(\sigma^{2} \mathrm{D} / \sigma^{2} \mathrm{~A}\right)^{1 / 2}$, was observed to be more than 1 for all the characters which pointed the gene action towards over-dominance nature. These results are in accordance with Basal and Turgut (2005); Basal (2009); Imran et al. (2012) and Tang and Xiao (2013). Subhan et al. (2003); Pushpam and Raveendran (2005); Ali et al. (2011); Sarwar et al. (2011); Ali et al. (2015) indicated the preponderance of non-additive genetic effects for seed cotton yield attributes and fibre quality traits. Yuan et al (2001) and Ashraf and Ahmad (2000) advocated additive gene action owing to higher GCA variances.

The genotype BH-160 may be used in breeding programs due to its favorable general combining ability for most of the traits. Among male parents CIM-496 was considered desirable parent due to its good general combining ability for boll weight, seed mass per boll, lint mass per boll and seed cotton per plant. CIM-70 was considered good general combiner for seed volume, seed surface area and fibre fineness while S-12 showed the best GCA effects for seeds per boll, fibre length and fibre strength. These genotypes could be used in breeding programs for the improvement in respective traits.

Although, three way crosses like $(\mathrm{NIAB}-228 \times \mathrm{BH}-$ 160) $\times$ Reshmi-90; (NIAB-228 $\times$ BH-160) $\times$ S-12 and $(\mathrm{NIAB}-228 \times \mathrm{BH}-160) \times \mathrm{CIM}-496$ exhibited good SCA effects, yet they could not perform extra ordinary with respect to phenotype of the plant. Contribution of line $x$ tester interaction, to the total variability, was higher as compared to lines or testers individual contribution for all the characters which may be attributed to the preferences of parental genotypes to combine with each other.

Results of heterotic analysis revealed that the single crosses performed better as compared to the three way crosses with respect to various traits under discussion. NIAB-228 $\times$ NIAB-78 might be brought under consideration while attempting for improvement in traits like lint mass per boll, seed cotton per plant and fibre length through hybrid vigor. For increasing the average boll weight and seed density, the cross NIAB-228 $\times \mathrm{S}-12$ may be considered in future breeding programs while NIAB-228 $\times$ Reshmi-90 may be fruitful for increasing seed volume per 100 seeds and seed surface area. For improvement in fibre quality traits, three way crosses like $(\mathrm{NIAB}-228 \times \mathrm{BH}-160) \times \mathrm{S}-12$ and $(\mathrm{NIAB}-228 \times \mathrm{BH}-160)$ $\times$ Reshmi-90 may be useful due to their considerable heterotic percentage for the respective characters. Iqbal and Nadeem (2003); Khan et al. (2009); Zangi et al. (2009) and many other researchers reported heterosis for seed cotton yield and quality attributes in cotton.

An interesting, informative and worth notable fact from the current study was the relationship of seed number with other within-boll yield traits and the final seed cotton yield. Increase in seed mass per boll results either due to increase in seed number or seed volume. Heavier and bolder seeds, though preferable regarding 
germination and healthier seedlings, will exert an adverse effect on the lint mass per boll and lint percentage. On the other hand increased seed mass per boll due to increase in the number of medium sized seeds will result in amplified lint mass per boll and seed cotton per plant. This is because more the number of medium sized seeds, more will be the total surface area of seeds for greater lint production without affecting the lint mass per boll and lint percentage. This fact was evident in case of the cross NIAB-228 $\times$ NIAB-78, for which, increase in seed number per boll resulted in bigger lint mass per boll, total seed surface area (not on individual seed basis) and final seed cotton yield. It can also be noted that increase in the number of seeds per boll did not amplify the seed mass per boll significantly.

Correlation studies provide the information about type/direction and strength of association among various traits under study and with the economic yield of the crop. As has been well established, number of bolls and boll weight were the largest contributor to the seed cotton yield. Similar results have been reported by many researchers; Aguado et al. (2010); Azhar et al. (2004); Desalegn et al. (2009); Farooq et al. (2013); Chattha et al. (2013). Simultaneous improvement in seed cotton yield and fibre quality traits observed to be non-practical, as has been well established, due to negative association of fibre length, fibre strength and fibre fineness with seed cotton yield. Strong linkage and failure of independent assortment are the main causes of this negative relationship. Earlier reports by Yuan et al. (2001); Azhar et al. (2004) about negative correlation between yield and quality traits well supported the current results.

Considering association of within-boll yield components with agronomic and fibre quality traits revealed that, among within-boll yield components, seed number per boll, seed mass per boll and lint mass per boll were the main contributors to seed cotton yield in decreasing order $(56.9 \%, 42.8 \%$ and $31.5 \%$, respectively). Similar results were documented by Smith and Coyle (1997); Basal et al. (2009); Tang and Xiao (2014). Boll weight increased due to increase in seed number per boll, but not seed mass per boll, and lint mass per boll which also seems to be logical in the way that more the number of seeds, more will be the total surface area of seed for greater lint production, hence will increase the boll weight and ultimately the seed cotton and lint yield. Worley et al. (1974); Culp and Harrell (1975); Smith and Coyle (1997); Imran et al. (2012) also reported same results regarding number of seeds and seed mass per boll.

\section{CONCLUSIONS}

Within-boll yield components played a basic role in determination of seed cotton yield and fibre quality. All the parameters studied in present work showed the predominance of non-additive genetic control. Heterosis breeding could be rewarding when attempting to improve the within-boll yield components and fibre quality. Increase in seed mass per boll was the key factor for greater seed cotton yield but this increase should be in number rather than the size of the seed. Increase in number of seeds (of medium size) per boll will increase surface area per seed for greater lint production. On the other hand increase in seed mass per boll owing to the larger and bolder seeds will lower the lint mass per boll and ultimately lint percentage. Increased number of seeds per boll, seed mass per boll and lint mass per boll may be considered as effective selection criteria for improving within-boll yield components and ultimately the final yield.

\section{LITERATURE CITED}

Aguado, A., B.D.L. Santos, D. Gamane, L.G.D. Moral and F. Romero. 2010. Gene effects for cotton-fibre traits in cotton plant (Gossypium hirsutum L.) under Verticilliumconditions. Field Crops Res. 116: 209-217.

Ali, B., M.S. Iqbal, M.K.N. Shah, G. Shabbir and N.M. Cheema. 2011. Genetic analysis for various traits in Gossypium hirsutum L. Pak. J. Agri. Res. 24: 8-13.

Ali, I., A. Shakeel, A.A. Khan and B. Sadia. 2015. Genetic basis of variation for within-boll yield components in cotton. Turk J. Agri. For. DOI: 10.3906/tar-1409-117.

Ashraf, M. and S. Ahmad. 2000. Genetic effects for yield components and fibre characteristics in upland cotton (Gossypium hirsutum L.) cultivated under salinized $(\mathrm{NaCl})$ conditions. Agronomie, 20: 917-926.

Azhar, F.M., M. Naveed and A. Ali. 2004. Correlation analysis of seed cotton yield with lint characteristics in Gossypium hirsutum L. Int. J. Agri. Bio. 6: 656-658.

Bardak, A. and Y. Bolek. 2012. Genetic diversity of diploid and tetraploid cottons determined by SSR and ISSR markers. Turkish J. Field Crops. 17: 139-144.

Basal, H. and I. Turgut. 2005. Genetic analysis of yield components and fibre strength in upland cotton (Gossypium hirsutum L.). Asian J. Plant Sci. 4: 293-298.

Basal, H., A. Unay, O. Canavar and I. Yavas. 2009. Combining ability for fibre quality parameters and within-boll yield components in intraspecific and interspecific cotton populations. Spanish J. Agri. Res. 7: 364-374.

Bednarz, C.W., R.L. Nichols and S.M. Brown. 2006. Plant density modifications of cotton within-boll yield components. Crop Sci. 46: 2076-2080.

Bednarz, C.W., R.L. Nichols and S.M. Brown. 2007. Within-boll yield components of high yielding cotton cultivars. Crop Sci. 47: 2108-2112.

Chattha, W.S., J. Farooq, A. Ahmad, S.A. Kang and M.N. Haq. 2013. Correlation analysis of quality and yield contributing traits in upland cotton (Gossypium hirsutum L.). Int. J. Modern Agri. 2: 95-101.

Coyle, G.G. and C.W. Smith. 1997. Combining ability for within-boll yield components in cotton, Gossypium hirsutum L. Crop Sci. 37: 1118-1122.

Culp, T.W. and D.C. Harrell. 1975. Influence of lint percentage, boll size and seed size on lint yield of upland cotton with high fibre strength. Crop Sci. 15: 741-746.

Desalegn, Z., N. Ratanadilok and R. Kaveeta. 2009. Correlation and heritability for yield and fibre quality parameters of Ethiopian cotton (Gossypium hirsutum L.) estimated from 15 diallel crosses. Kasetsart J. Nat. Sci. 43: 1-11.

Farooq, J., M. Anwar, M. Riaz, A. Mahmood, A. Farooq and M.S. Iqbal. 2013. Association and path analysis of earliness yield and fibre related traits under cotton leaf curl virus $(\mathrm{CLCuV})$ intensive conditions in Gossypium hirsutum L. Plant Knowledge J. 2: 43-50. 
Green, C. and T.W. Culp. 1990. Simultaneous improvement of yield, fibre quality and yarn strength in upland cotton. Crop Sci. 30: 66-69.

Hodson, E.A. 1920. Lint frequency in cotton with a method for determination. pp. 3-11. Ark. Agri. Exp. Sta. Bul. No. 168.

Imran, M., A. Shakeel, F.M. Azhar, J. Farooq, M.F. Saleem, A. Saeed, W. Nazeer, M. Riaz, M. Naeem and A. Javaid. 2012. Combining ability analysis for within-boll yield components in upland cotton (Gossypium hirsutum L.). Genet. Mol. Res. 11: 2790-2800.

Iqbal, M.Z. and M.A. Nadeem. 2003. Generation mean analysis for seed cotton yield and number of sympodial branches per plant in cotton (Gossypium hirsutum L.). Asian J. Plant Sci. 2: 395-399.

Kempthorne, O. 1957.An introduction to genetics statistics.New York, John Wiley and Sons Inc. London.

Khan, N.U., G. Hassan, M.B. Kumbhar, K.B. Marwat, M.A. Khan, A. Parveen and M. Saeed. 2009. Combining ability analysis to identify suitable parents for heterosis in seed cotton yield, its components and lint $\%$ in upland cotton. Indust. Crops and Prod. 29: 108-115.

Pakistan Economic Survey.2012-13. Government of Pakistan.Ministry of Finance, Economic Advisors Wing, Islamabad.

Pearson, K. 1920. Notes on history of correlation. Biometrika 13: $25-45$

Pole, S., S. Kamble, I. Madrap and D. Sarang. 2008. Diallel analysis for combining ability for seed cotton yield and its components in upland cotton (Gossypium hirsutum L.). J. Cotton Res. Develop. 22: 19-22.

Preetha, S. and T. Raveendran. 2008. Combining ability and heterosis for yield and fibre quality traits in line $\times$ tester crosses of upland cotton (Gossypium hirsutum L.). Int. J. Plant Breed. Genet. 2: 64-74.

Pushpam, R. and T.S. Raveendran. 2005. Heterosis and combining ability studies in upland cotton for fibre characters. Tropical Agric. Res. Ext. 8: 65-70.
Sarwar, G., M. Baber, N. Hussain, I.A. Khan, M. Naeem, M. Amanullah and A.A. Khan. 2011. Genetic dissection of yield and its components in upland cotton (Gossypium hirsutum L.). African J. Agri. Res. 6: 2527-2531.Shakeel, A., F.M. Azhar and I.A. Khan. 2008. Assessment of earliness in Gossypium hirsutum L. Pak. J. Agri. Sci. 45: 80-87.

Smith, C.W. and C.G. Coyle. 1997. Association of fibre quality parameters and within-boll yield components in upland cotton. Crop Sci. 37: 1775-1779.

Subhan, M., M. Qasim, R.D. Ahmad, M.U. Khan, M.A. Khan and M.A. Amin. 2003. Diallel analysis for estimating combining ability of quantitatively inherited traits in upland cotton. Asian J. Plant Sci. 2: 853-857.

Swamy, M.L., M. Gopinath, K. Gopala and K. Murthy. 2013. Line $\times$ tester analysis for yield and yield attributes in upland cotton (Gossypium hirsutum L.). Helix. 5: 378-382.

Tang, F. and W. Xiao. 2013. Genetic effects and heterosis of within-boll yield components in upland cotton (Gossypium hirsutum L.). Euphytica. 194: 41-51.

United States Department of Agriculture. Foreign Agriculture Service. World Agricultural Production. Circular series WAP 12-13. December, 2013.

Worley, S., T.W. Culp and D.C. Harrell. 1974. The relative contributions of yield components to lint yield of upland cotton, Gossypium hirsutum L. Euphytica. 23: 399-403.

Yuan, L., T. Zhang, W. Guo, J. Pan and R.J. Kohel. 2001. Heterosis and gene action of boll weight and lint percentage in high quality fibre property varieties in upland cotton. Zuowuxuebao. 28: 196-202.

Zangi, M.R., N.B. Jelodar, S.K. Kazemitabar and M. Vafaeitabar. 2009. Cytoplasmic and combining ability on fibre quality traits in intra and inter-specific crosses of tetraploid cotton (G. hirsutum L. $\times$ G. barbadense L.). American-Eurasian J. Agri. Env. Sci. 5: 519-525. 